\title{
Three-dimensional Information and Refractive Index Measurement Using a Dual-wavelength Digital Holographic Microscope
}

\author{
Sanghoon Shin \\ AP \& Tec, Suseo Dong, Kangnam Gu, Seoul 135-539, Korea \\ Younghun Yu* \\ Department of Physics, Cheju National University, Jeju 690-756, Korea
}

(Received March 23, 2009 : revised May 7, 2009 : accepted June 9, 2009)

\begin{abstract}
Digital holographic microscopy allows optical path difference measurement. Optical path difference depends on the both refractive index and morphology of sample. We developed a dual-wavelength in-line digital holographic microscope that can measure simultaneously the refractive index and morphology of a sample, providing highly precise three-dimensional information. Here we propose theoretical and experimental methods for dual-wavelength in-line digital holographic microscopy. The measured data were reasonable, although there was data error. By improving the experimental method, we could measure the refractive index more precisely and obtain more accurate threedimensional information on samples.
\end{abstract}

Keywords: Holography, Image reconstruction, Diffractive optics

OCIS codes : (090.0090) Holography; (100.3010) Image reconstruction; (050.1970) Diffractive optics

\section{INTRODUCTION}

Holography is a method of recording phase modulation by using the light reflected or transmitted from a projected object onto a photoplate in the form of interference patterns. Object and reference beams are required for the recording, and an interference pattern is generated from the combination of the two beams. In the past, the interference patterns were recorded on film plates, but now charge-coupled devices (CCDs) or complementary metal-oxide semiconductor cameras are normally used, and a computer is used to reconstruct the hologram.

Yaroslavskii and his colleague proposed a method of numerical hologram reconstruction in the 1970s, and Ounral and Scott used numerical reconstruction to measure the size of a particle after improving on the reconstruction algorithm [1-6]. This method of digitally recording and reconstructing a numerical hologram is known as digital holography [7-10]. Digital holography has many advantages. For example, it does not require any chemical

*Corresponding author: yyhyoung@cheju.ac.kr processing because the reconstructed image can be easily observed on a computer monitor, and experts can obtain numerical data for three-dimensional (3-D) objects [11,12].

In the reconstruction process, the refractive index of the sample is either known or assumed to be homogeneous. Practically, the phase shift, or optical path difference induced from the difference in refractive index between the sample and the surrounding medium and is proportional to the thickness of the sample. If the refractive index of the sample is unknown or not homogeneous, the reconstructed 3-D image will not be identical to the original sample [8]. This is a limitation of digital holography. If one measures the refractive index using digital holography, one can determine the $3-\mathrm{D}$ shape of the sample $[13,14]$.

In this study, we constructed a dual-wavelength in-line digital holographic microscope system for measuring the refractive index of a sample. After measuring some holograms, we calculated the refractive index of the samples using the phase difference between the holograms. The measured indexes represented the refractive values and refractive index distributions of the sample. 


\section{THEORETICAL MODEL}

\section{Hologram recording and reconstruction}

In the hologram recording process, a plane reference wave $(R)$ and a diffusively reflected object wave $(O)$ interfere at the CCD. The hologram intensity is given by

$$
I_{H}(x, y)=|R|^{2}+|O|^{2}+R^{*} O+R O^{*},
$$

where $R^{*}$ and $O^{*}$ denote the complex conjugates of the reference and object waves, respectively [1,2]. The digital holographic image can be recorded using a black-andwhite CCD camera. The digital

hologram $I_{H}(k . l)$ is an $N \times N$ array resulting from the two-dimensional sampling of $I_{H}(x, y)$ by the CCD camera. It is given by

$$
I_{H}(k, l)=I_{H}(x, y) \operatorname{rect}\left(\frac{x}{L}, \frac{y}{L}\right) \times \sum_{k=-N / 2}^{N / 2} \sum_{\sum=-N / 2}^{N / 2} \delta(x-k \Delta x, y-l \Delta y),
$$

where $K$ and $l$ are integers, $L \times L$ denotes the area of the CCD chip, and $\Delta x$ and $\Delta y$ indicate the pixel size of the CCD.

In classical optical holography, the object wave can be reconstructed by illuminating the processed hologram with a plane wave similar to that used in the recording process. Looking through the hologram, one observes a virtual image. If a screen is placed at a distance $d$ behind the hologram, a real image is formed on it. Mathematically, the amplitude and phase distributions in the plane of the real image can be found using the FresnelKirchhoff integral $[1,2]$. If a plane wave illuminates the hologram with an amplitude transmittance $I_{H}(x, y)$, the Fresnel-Kirchhoff integral yields a complex amplitude $\Psi(\xi, \eta)$ in the real image plane

$$
\begin{aligned}
& \Psi(\xi, \eta)=\frac{\exp (i 2 \pi d / \lambda)}{i \lambda d} \exp \left[\frac{i \pi}{d \lambda}\left(\xi^{2}+\eta^{2}\right)\right] \times \iint I_{H}(x, y) \exp \left[\frac{i \pi}{\lambda d}\left(x^{2}+y^{2}\right)\right] \\
& \times \exp \left[\frac{i 2 \pi}{\lambda d}(\xi x+\eta y)\right] d x d y,
\end{aligned}
$$

where $\lambda$ is the wavelength and $d$ is the reconstruction distance. From Eq. (3), the Fresnel-Kirchhoff integral can be considered the Fourier transformation of the function $I_{H}(x, y) \exp \left[i \pi\left(x^{2}+y^{2}\right) / \lambda d\right]$ at the spatial frequencies $\xi$ and $\eta$. Because $\Psi(\xi, \eta)$ is an array of complex numbers, one can obtain an amplitude-contrast image using the intensity

$$
I(\xi, \eta)=\operatorname{Re}[\Psi(\xi, \eta)]^{2}+\operatorname{Im}[\Psi(\xi, \eta)]^{2} .
$$

The phase-contrast image is obtained by calculating

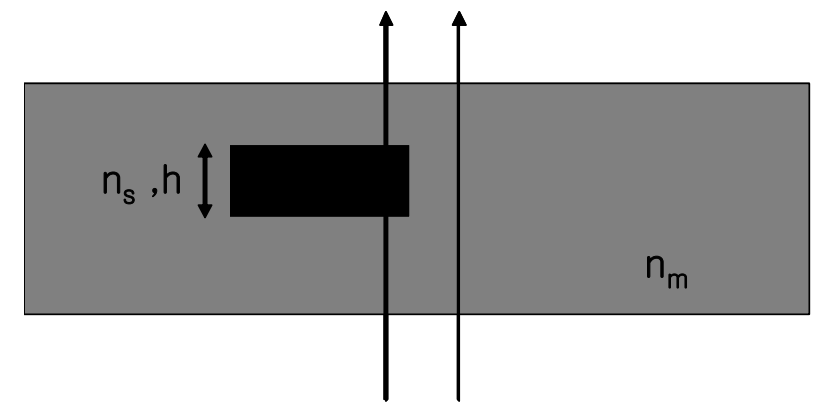

FIG. 1. Optical path difference between samples with different refractive indexes.

the argument

$$
\psi(\xi, \eta)=\arctan \left\{\frac{\operatorname{Im}[\Psi(\xi, \eta)]}{\operatorname{Re}[\Psi(\xi, \eta)]}\right\} .
$$

The real three-dimensional information is acquired by phase unwrapping with phase contrast image.

\section{Measurement of the refractive index using a dual- wavelength hologram}

Consider the optical path difference of a beam traveling through the transparent sample along the arrow in Fig. 1:

$$
O P D(x, y)=\left(n_{s}(x, y)-n_{m}(x, y)\right) h(x, y) .
$$

The phases with dual wavelengths are given by

$$
\begin{aligned}
& \phi_{1}\left(x, y ; \lambda_{1}\right)=\frac{2 \pi}{\lambda_{1}} \bullet O P D\left(x, y, \lambda_{1}\right) \\
& \phi_{2}\left(x, y ; \lambda_{1}\right)=\frac{2 \pi}{\lambda_{2}} \bullet O P D\left(x, y, \lambda_{2}\right) .
\end{aligned}
$$

If $n_{m}$ and $n_{s}$ remain almost the same between two wavelengths and one refractive index $n_{m}$ or $n_{s}$ is known at two wavelengths, one can calculate simultaneously the average of the other refractive index and the height.

The refractive index of sample is given by

$$
n_{s}(x, y)=\frac{n_{m}\left(\lambda_{1}\right)+n_{m}\left(\lambda_{2}\right) \frac{\lambda_{1} \phi_{1}}{\lambda_{2} \phi_{2}}}{\frac{\lambda_{1} \phi_{1}}{\lambda_{2} \phi_{2}}-1}
$$

To calculate the refractive index of the sample, the dual wavelength hologram recording is performed sequentially. The concept of recording two holograms at different wavelength has been demonstrated and is proposed to solve $2 \pi$ ambiguities in digital holography [15-18]. We can calculate the distribution of refractive indices of the sample simultaneously using dual-wavelength digital holography. This is a merit of dual-wavelength digital holo- 


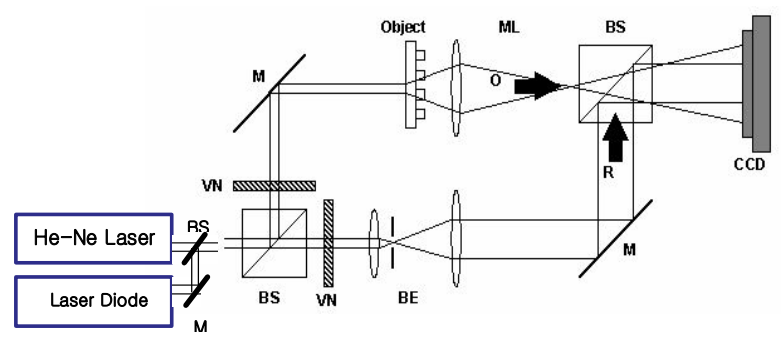

FIG. 2. Transmission-type dual-wavelength digital holographic microscope. VN: variable-density filter; BS: beam splitter; M: mirror; O: object wave; R: reference wave; $\mathrm{BE}$ : beam expander with spatial filter; ML: microscope objective lens; CCD: charge-coupled device.

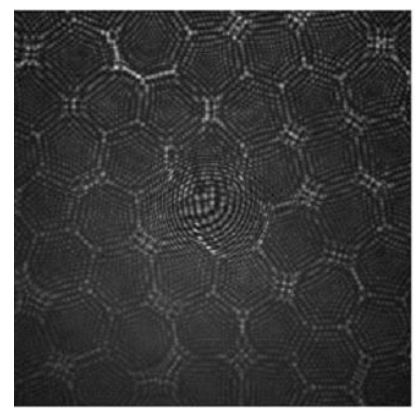

(a)

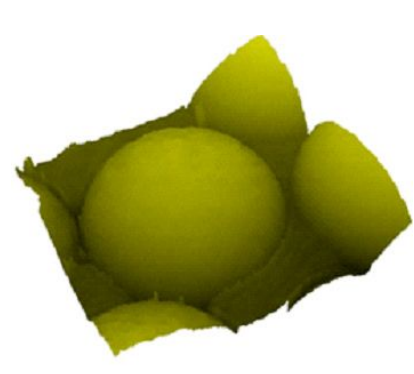

(c)

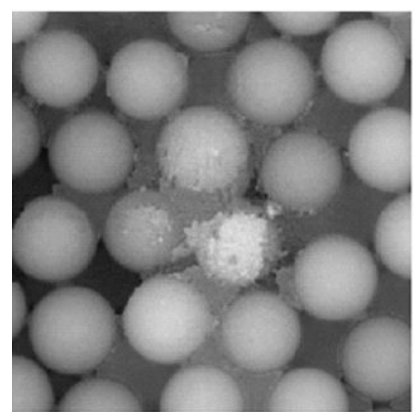

(e)

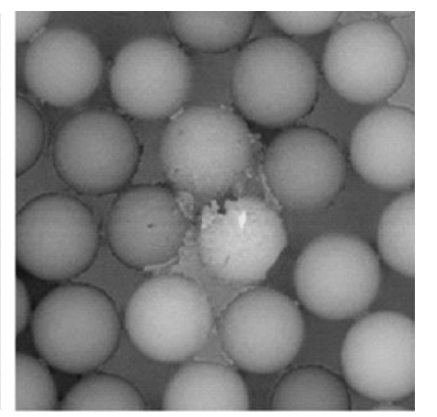

(b)

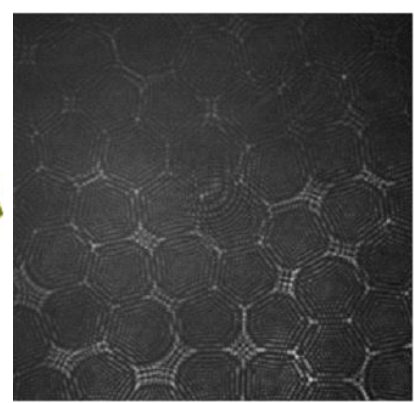

(d)

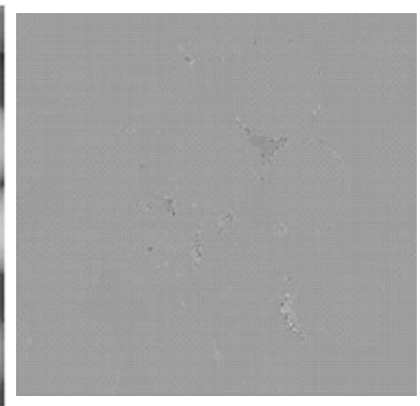

(f)
FIG. 3. Dual-wavelength holography and refractive index of a micro-lens array. (a), (d) Hologram created using HeNe laser (632 nm) and laser diode (532 nm). (b), (e) Phase unwrapping grayscale image of holograms (a) and (d), respectively. (c) Three-dimensional image and (f) grayscale of refractive index. graphy. Usually, scanning processes are necessary to measure the refractive indices distribution on the sample in previous work. To calculate the phase of the same point from a hologram with a different wavelength, one should resize the holograms [19-20].

\section{EXPERIMENTAL SETUP AND RESULTS}

Figure 2 shows a schematic of a dual-wavelength transmission holographic microscope. The basic experimental setup is similar to that of a Mach-Zehnder-type interferometer. A 10-mW He-Ne laser and 532-nm laser diode were used as the light source, and the objective lens of a microscope (ML) was used to expand the beam passing through the sample. We used neutral-density filters (VN) to obtain the interference patterns for maximum contrast. A beam expander (BE) was used for the reference beam in the $\mathrm{TEM}_{00}$ mode. We used a CCD camera (Sony IPX4M15L) to record the holograms. The pixel size and the number of pixels were $7.4 \mu \mathrm{m} \times 7.4$ $\mu \mathrm{m}$ and $2048 \times 2048$, respectively. The CCD was placed $20 \mathrm{~cm}$ from the ML, and the overlapping angle between the reference and objective rays was maintained at $0^{\circ}$, resulting in in-line holography.

Figure 3 shows the experimental results of a micro-lens array. The height and diameter of micro-lens are $50 \mu \mathrm{m}$ and $30 \mu \mathrm{m}$, respectively. The refractive index of this lens, which was made of polyethylene terephthalate, was 1.575 at the 550-nm wavelength. The index matching oil was used for reference $\left(n_{m}\right)$. Its refractive index was 1.526 at the $632-\mathrm{nm}$ wavelength and 1.535 at the $532-\mathrm{nm}$ wavelength. We used index matching oil to resolve the $2 \pi$ ambiguity, because the height of the sample was greater than that of the wavelength $[17,18]$. If the height of the sample had been less than the wavelength, the matching oil would not have been necessary.

Figures 3(a) and (d) are holograms created by the $\mathrm{He}-\mathrm{Ne}$ laser $(632 \mathrm{~nm})$ and laser diode (532 nm), respectively. To calculate the optical path difference by refractive index of the micro-lens array, we constructed a 3-D image using phase unwrapping. Figures 3(b) and (e) are the 3-D gray-level images of holograms (a) and (d). Resizing was necessary to calculate the phase of the same point from a hologram of different wavelength. Remember that dual-wavelength digital holography measures the refractive index of a plane rather than a point in the sample. We calculated the refractive index of the micro-lens using Eqs. (7) and (8). Figure 3(f) shows the calculated gray-level refractive index image. As shown in Fig. 3(f), the gray levels were almost the same, which means that the whole region had the same refractive index. This result is reasonable, because the micro-lens array was made from one material. Although the measured value of 1.56 was different from the manufacturer's specification of 1.575, dual-wavelength digital holography 


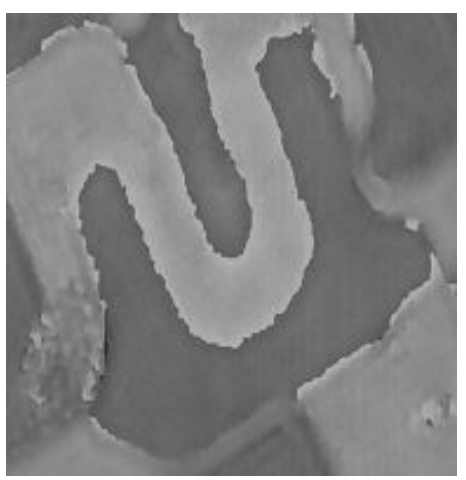

(a)

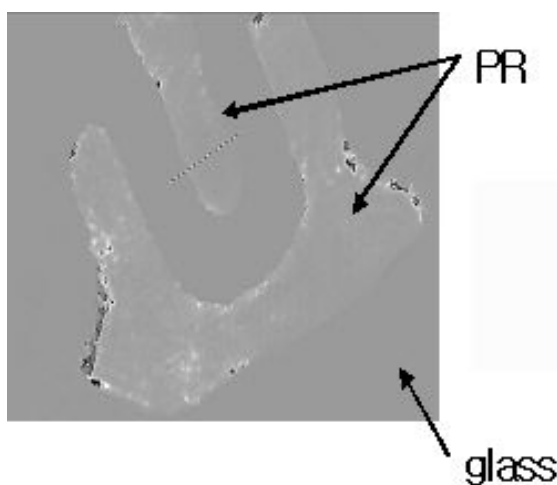

(c)

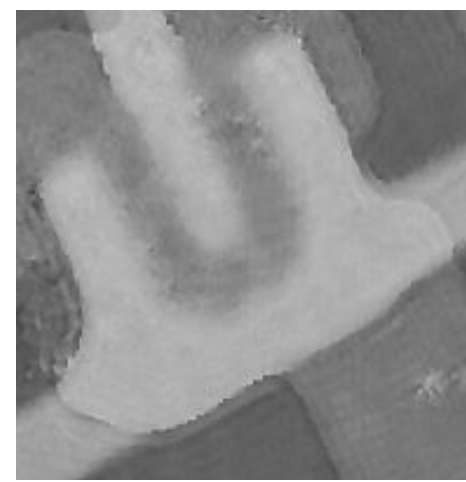

(b)

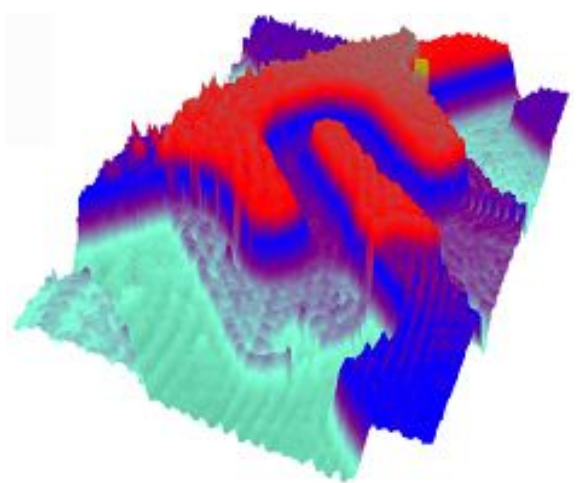

(d)

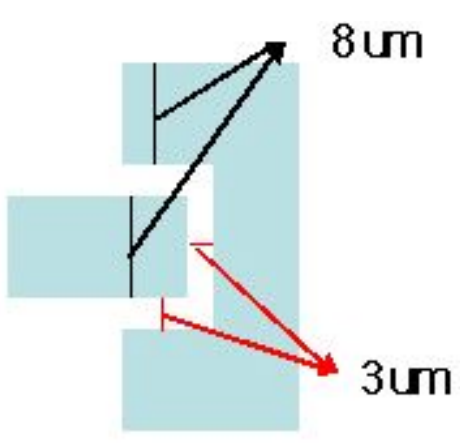

(e)

FIG. 4. Dual-wavelength holography and refractive index of a thin film transistor on glass. (a), (b) Phase unwrapping image of hologram created using a He-Ne laser $(632 \mathrm{~nm})$ and laser diode $(532 \mathrm{~nm})$, respectively. (c) gray level of refractive index and (d) 3-D image, (e) design of TFT.

is applicable to measurements of the refractive index. Figure 3(c) shows a 3-D image of the micro-lens.

Figure 4 shows experimental results for the thin film transistor on glass. The pattern was fabricated by a photo-resistor (PR). The refractive index of the PR and glass were 1.68 and 1.54, respectively. Figures 4(a) and (b) show the phase unwrapping image of the hologram created using dual-wavelength digital holography, where (a) is the result of the $\mathrm{He}-\mathrm{Ne}$ laser (632 nm) and (c) the laser diode (532 nm). Figure 4(d) is the 3-D image of the thin film transistor on the glass. Figure 4(c) shows the calculated refractive index using phase unwrapping results (a) and (b). As shown in Fig. 4(c), the gray levels were different for the glass and PR regions. This is a result of the difference in the refractive indexes of $\mathrm{PR}$ and glass. The calculated average refractive indexes of $\mathrm{PR}$ and glass were 1.64 and 1.52, which were in reasonable agreement with actual values. From these results, we confirmed that dual-wavelength digital holography is applicable to refractive index and 3-D shape measurement.

\section{CONCLUSIONS}

Digital holographic microscopy can be used to measure optical path differences with a high degree of accuracy. Optical path differences depend on the refractive index and morphology of the samples. To acquire 3-D information on a sample, one must know either the refractive index or the morphology. We have developed a dual-wavelength digital holographic microscope that can measure the refractive index and morphology separately. And we can calculate the distribution of refractive indices of the sample simultaneously using dual-wavelength digital holography. This is a merit of dual-wavelength digital holography. Usually, scanning processes are necessary to measure the refractive indices distribution on the sample in previous work. This system is applicable to measure the refractive index value and distribution of sample.

\section{ACKNOWLEDGMENT}

This study was supported by the Korean Ministry of Commerce, Industry, and Energy. A number of the researchers participating in this study were supported by a grant from the "Second-phase BK21 project." 


\section{REFERENCES}

1. E. Cuche, P. Marquet, and C. Depeursinge, "Simultaneous amplitude-contrast and quantitative phase-contrast micros copy by numerical reconstruction of Fresnel off-axis holograms,” Appl. Opt. 38, 6994-7001 (1999).

2. L. Xu, X. Peng, Z. Guo, J. Miao, and A. Asundi, "Studies of digital microscopic with application to microstructure testing," Appl. Opt. 40, 5046-5051 (2001).

3. M. Jeong, N. Kim, and J. H. Park, "Elemental image synthesis for integral imaging using phase-shifting digital holography,” J. Opt. Soc. Korea 12, 275-280 (2008).

4. M. A. Kronrod, N. S. Merzlyakov, and L. P. Yaroslavski, "Reconstruction of hologram with a computer," Sov. Phys. Tech. 17, 434-444 (1972).

5. L. P. Yaroslavskii and N. S. Merzlyakov, Methods of Digital Holography (Consultants Bureau, New York, USA, 1980).

6. L. Onural and P. D. Scott, "Digital decoding of in-line holograms,” Opt. Eng. 26, 1124-1132 (1987).

7. J. W. Goodman, Introduction to Fourier Optics, 2nd ed., edited by J. Goodman (McGraw Hill, New York, USA, 2005), Chapter 9.

8. U. Schnars and W. Juepther, Digital Holography, edited by U. Schnars and W. Juepther (Springer, Heidelberg, Germany, 2005).

9. L. Xu, J. Miao, and A. Asundi, "Properties of digital holography based on in-line configuration," Opt. Eng. 39, 3214-3219 (1999).

10. C. Depeursinge, Digital Holography and Three-dimensional Display, edited by T. C. Poon (Springer, New York, USA, 2006).

11. D. Kim, B. J. Baek, Y. D. Kim, and B. Javidi, "3D nano object recognition based on phase measurement technique," J. Opt. Soc. Korea 11, 108-112 (2007).

12. J. W. Kang and C. K. Hong, "Three dimensional shape measurement of a micro Fresnel lens with in-line phaseshifting digital holographic microscopy," J. Opt. Soc. Korea 10, 178-183 (2006).

13. H. Cho, J. Lim, D. C. Kim, S. Shin, and Y. Yu, "Threedimensional information and two-dimensional refractive index measurements using combined digital holographic microscope," J. Korean Phys. Soc. 53, 2391-2395 (2008).

14. J. Kühn, F. Charrièrea, T. Colomba, E. Cuche, Y. Emery, and C. Depeursinge, "Measurements of Corner cubes microstructures by high-magnification digital holographic microscopy," Proc. SPIE 6188, 618804-618814 (2006).

15. C. Polhemus, "Two-wavelength interferometry," Appl. Opt. 12, 2071-2074 (1973).

16. J. Gass, A. Dako, and M. K. Kim, "Phase imaging without 2 pi ambiguity by multiwavelength digital holography," Opt. Lett. 28, 1141-1143 (2003).

17. Y. Fu, G. Pedrini, B. Hennelly, R. Groves, and W. Osten, "Dual-wavelength image-plane digital holography for dynamic measurement," Opt. Laser Technol. 47, 552-557 (2008)

18. K. Creath, Y. Cheng, and J. C. Wyant, "Contouring aspheric surfaces using two-wavelength phase-shifting interferometry," Optica Acta 32, 1455-1464 (1985).

19. P. Ferraro, S. D. Nicola, G. Coppola, A. Finizio, D. Alfieri, and G. Pierattini, "Controlling image size as a function of distance and wavelength in Fresnel-transform reconstruction of digital holograms," Opt. Lett. 29, 844846 (2004).

20. N. Demoli, D. Vukicevic, and M. Torzynski, "Dynamic digital holographic interferometry with three wavelengths," Opt. Exp. 11, 767-774 (2003). 